Pacific Journal of Mathematics

BIHOLOMORPHIC MAPPINGS BETWEEN WEAKLY 


\title{
BIHOLOMORPHIC MAPPINGS BETWEEN WEAKLY PSEUDOCONVEX DOMAINS
}

\author{
JOHN ERIK FORNAESS
}

\begin{abstract}
Assume we have a biholomorphic mapping between weakly pseudoconvex domains. It is an old question whether this extends to a diffeomorphism between their closures. The well known theorem of Fefferman states that this is true for strongly pseudoconvex domains. We will show that if the map has a smooth extension to the boundary, then it cannot map an analytic disc in the boundary to a single point.

This is an immediate consequence of the following theorem.
\end{abstract}

THEOREM. Assume $\Omega, W$ are bounded pseudoconvex sets with $\mathscr{C}^{2}$ boundary in $\boldsymbol{C}^{n}$, and assume $\Phi: \Omega \rightarrow W$ is a biholomorphic map with a $\mathscr{C}^{2}$-extension $\Phi: \bar{\Omega} \rightarrow \bar{W}$. Then $\Phi$ is a $\mathscr{C}^{2}$-diffeomorphism between $\bar{\Omega}$ and $\bar{W}$.

This theorem generalizes a similar result for strongly pseudoconvex domains by the author [2], see also Pinchuk [3].

The theorem is false in general for $\mathscr{C}^{1}$-domains and maps $\Phi$ with $\mathscr{C}^{1}$-extensions. To see this, let $\Omega=\{z \in C ;|z+1|<1\}$ and let $\Phi(z)=$ $z / \log z$ and $W=\Phi(\Omega)$.

Also the theorem is false in general for proper holomorphic maps. For example, let $\Omega=\left\{(z, w) \in C^{2} ;|z|^{2}+|w|^{4}<1\right\}, W=\left\{(z, w) ;|z|^{2}+\right.$ $\left.|w|^{2}<1\right\}$ and $\Phi(z, w)=\left(z, w^{2}\right)$.

Proof of the Theorem. Let us fix a point $p \in b \Omega$. We want to show that $\Phi^{\prime}(p)$ is a nonsingular linear transformation. The proof is complete if this is true for all $p$ in the boundary of $\Omega$.

To simplify the computations, we will make affine complex changes of coordinates such that $p$ becomes the origin in $\boldsymbol{C}^{n}$ with variables $\left(z_{1}, \cdots, z_{n}\right)$ and $\Phi(p)$ becomes the origin in $C^{n}$ with variables $\left(w_{1}, \cdots, w_{n}\right)$. We may arrange this such that

$$
\begin{aligned}
\Omega & =\left\{z=\left(z_{1}, \cdots, z_{n}\right) ; \rho(z)=\operatorname{Re} z_{1}+R\left(z_{1}, \cdots, z_{n}\right)<0\right\} \text { and } \\
W & =\left\{w=\left(w_{1}, \cdots, w_{n}\right) ; \sigma(w)=\operatorname{Re} w_{1}+S\left(w_{1}, \cdots, w_{n}\right)<0\right\}
\end{aligned}
$$

where $R, S$ are $\mathscr{C}^{2}$-functions which vanish to at least second order at the origin.

From Diederich, Fornaess [1] it follows that there exists a $\mathscr{C}^{2}$ defining function $\hat{\sigma}$ of $W$ such that $-(-\hat{\sigma})^{2 / 3}$ is strictly plurisubharmonic in $W$ near the origin. It follows that $-(-\hat{\sigma})^{2 / 3} \circ \Phi$ is strictly 
plurisubharmonic in $\Omega$ near the origin.

We apply the Hopf lemma to points in $\Omega$ of the form $(t, 0, \cdots, 0)$ with $-1 \ll t<0$. There must exist a $K>0$ such that

$$
-(-\hat{\sigma})^{2 / 3}(\Phi(t, 0, \cdots, 0)) \leqq K t .
$$

Since $\sigma$ is of the same order of magnitude as $\hat{\sigma}$, we obtain for a possibly different $K$, that

$$
-\operatorname{Re} \varphi_{1}(t, 0, \cdots, 0)-S(\Phi(t, 0, \cdots, 0)) \geqq K|t|^{3 / 2}
$$

where we have written $\Phi=\left(\varphi_{1}, \cdots, \varphi_{n}\right)$. Hence $\left(\partial \varphi_{1} / \partial z_{1}\right)(0, \cdots, 0)>0$.

Consider the $\mathscr{C}^{2}$-map $\Lambda\left(z_{1}, \cdots, z_{n}\right)=\left(\varphi_{1}\left(z_{1}, \cdots, z_{n}\right), z_{2}, \cdots, z_{n}\right)$. Then $\Lambda^{\prime}(0)$ is invertible. Therefore $\Lambda$ maps the germ of $\Omega$ at the origin to the germ of a pseudoconvex set $U$ with $\mathscr{C}^{2}$ boundary at the origin. We can describe $U$ by

$$
U=\left\{\eta=\left(\eta_{1}, \cdots, \eta_{n}\right) ; \tau(\eta)=\operatorname{Re} \eta_{1}+T\left(\eta_{1}, \cdots, \eta_{n}\right)<0\right\}
$$

for some $\mathscr{C}^{2}$-function $T$ vanishing to at least second order at the origin.

We will study the map $\Psi=\Phi \circ \Lambda^{-1}, \Psi=\left(\psi_{1}, \cdots, \psi_{n}\right)$. It suffices to show that $\Psi^{\prime}(0)$ is a nonsingular linear map. We can describe $\Psi$ by

$$
\Psi\left(\eta_{1}, \cdots, \eta_{n}\right)=\left(\eta_{1}, \psi_{2}\left(\eta_{1}, \cdots, \eta_{n}\right), \cdots, \psi_{n}\left(\eta_{1}, \cdots, \eta_{n}\right)\right) .
$$

If $\Psi^{\prime}(0)$ is singular, then we may assume, after a linear change in the $\left(w_{2}, \cdots, w_{n}\right)$ - and $\left(\eta_{2}, \cdots, \eta_{n}\right)$ - direction, that

$$
\Psi^{\prime}(0)=\left[\begin{array}{ccccr}
1 & 0 & 0 & \cdots & 0 \\
\frac{\partial \psi_{2}}{\partial \eta_{1}}(0) & 0 & \frac{\partial \psi_{2}}{\partial \eta_{3}}(0) & \cdots & \frac{\partial \psi_{2}}{\partial \eta_{n}}(0) \\
\vdots & \vdots & \vdots & & \\
\frac{\partial \psi_{n}}{\partial \eta_{1}}(0) & 0 & \frac{\partial \psi_{n}}{\partial \eta_{3}}(0) & \cdots & \frac{\partial \psi_{n}}{\partial \eta_{n}}(0)
\end{array}\right]
$$

Next we consider points $t_{1}=(t, 0, \cdots, 0)$ and $t_{2}=(t, t, 0, \cdots, 0)$ in $U$ with $-1 \ll t<0$. We then have the estimates, for $\tau_{i}=\Psi\left(t_{i}\right)$, $i=1,2$ :

$$
\tau_{i}=\left(t, \frac{\partial \psi_{2}}{\partial \eta_{1}}(0) \cdot t+0\left(t^{2}\right), \cdots, \frac{\partial \psi_{n}}{\partial \eta_{1}}(0) \cdot t+0\left(t^{2}\right)\right)
$$

$i=1,2$.

Define $W_{t}$ to be the set

$$
W_{t}=\left\{\left(w_{1}, \cdots, w_{n}\right) \in W ; w_{1}=t\right\} .
$$

There exists some $\delta>0$ independent of $t$ such that 


$$
W_{t} \supset\left\{\left(t, w_{2}, \cdots, w_{n}\right) ;\left\|\left(w_{2}, \cdots, w_{n}\right)\right\|<\delta|t|^{1 / 2}\right\}=\widetilde{W}_{t} .
$$

Let us write $\Phi^{-1}: W \rightarrow \Omega$ as $\Phi^{-1}=\left(\mu_{1}, \cdots, \mu_{n}\right)$. We then have that for some constant $K>0$, independent of $t$, that

$$
\left|\mu_{2}\left(t, w_{2}, \cdots, w_{n}\right)\right| \leqq K
$$

for all points in $\widetilde{W}_{t}$, since $\Omega$ is bounded. The points $\tau_{1}, \tau_{2}$ are in $\widetilde{W}_{t}$ and satisfy the estimates $\left\|\tau_{1}\right\| \leqq K_{1}|t|,\left\|\tau_{2}\right\| \leqq K_{1}|t|$ and $\left\|\tau_{1}-\tau_{2}\right\| \leqq$ $K_{1}|t|^{2}$ for some constant $K_{1}$ independent of $t$. It follows from Schwarz's lemma that for some constant $K_{2}$, independent of $t$, we have

$$
\left|\mu_{2}\left(\tau_{1}\right)-\mu_{2}\left(\tau_{2}\right)\right| \leqq K_{2}|t|^{3 / 2}
$$

However, by construction we know that $\left|\mu_{2}\left(\tau_{1}\right)-\mu_{2}\left(\tau_{2}\right)\right|=|t|$ which is a contradiction for all small enough $|t|$.

\section{REFERENCES}

1. K. Diederich and J. E. Fornaess, Exhaustion functions and Stein neighborhoods for smooth pseudoconvex domains, Proc. Nat. Acad. Sci. USA, 72 (1975), 3279-3280.

2. J. E. Fornaess, Embedding strictly pseudoconvex domains in convex domains, Amer.

J. Math., 98 (1976), 529-569.

3. S. I. Pinchuk, On proper holomorphic mappings of strictly pseudoconvex domains, Siberian Math. J., (1975), 644-649 (translated from Sibirskii Mat. Zhurnal, 15 (1974), 909-917).

Received May 12, 1977.

PRINCETON UNIVERSITY

PRINCETON, NJ 08540 



\section{PACIFIC JOURNAL OF MATHEMATICS}

\section{EDITORS}

RICHARD ARENS (Managing Editor)

University of California

Los Angeles, California 90024

C. W. Curtis

University of Oregon

Eugene, OR 97403

C. C. MOORE

University of California

Berkeley, CA 94720

\section{J. DUGUNDJI}

Department of Mathematics University of Southern California Los Angeles, California 90007

R. Finn AND J. Milgram Stanford University Stanford, California 94305

ASSOCIATE EDITORS
E. F. BECKENBACH

B. H. NeUMANN

F. WOLF

K. YoshidA

\section{SUPPORTING INSTITUTIONS}

UNIVERSITY OF BRITISH COLUMBIA UNIVERSITY OF SOUTHERN CALIFORNIA CALIFORNIA INSTITUTE OF TECHNOLOGY STANFORD UNIVERSITY UNIVERSITY OF CALIFORNIA MONTANA STATE UNIVERSITY UNIVERSITY OF TOKYO UNIVERSITY OF NEVADA, RENO UNIVERSITY OF UTAH NEW MEXICO STATE UNIVERSITY OREGON STATE UNIVERSITY UNIVERSITY OF OREGON

WASHINGTON STATE UNIVERSITY UNIVERSITY OF WASHINGTON OSAKA UNIVERSITY 


\section{Pacific Journal of Mathematics}

\section{Vol. 74, No. $1 \quad$ May, 1978}

Gerald Arthur Anderson, Computation of the surgery obstruction groups

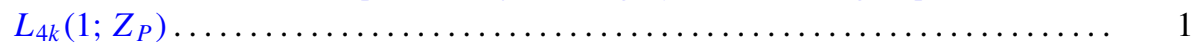

R. K. Beatson, The degree of monotone approximation ................ 5

Sterling K. Berberian, The character space of the algebra of regulated functions . . . 15

Douglas Michael Campbell and Jack Wayne Lamoreaux, Continua in the plane with

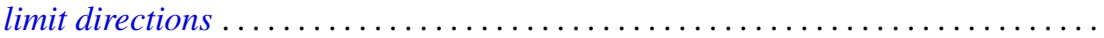

R. J. Duffin, Algorithms for localizing roots of a polynomial and the Pisot

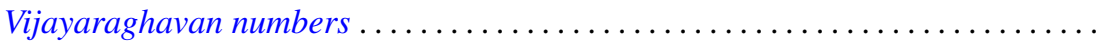

Alessandro Figà-Talamanca and Massimo A. Picardello, Functions that operate on

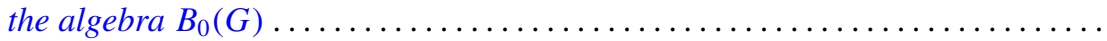

John Erik Fornaess, Biholomorphic mappings between weakly pseudoconvex

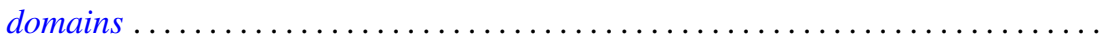

Andrzej Granas, Ronald Bernard Guenther and John Walter Lee, On a theorem of S.

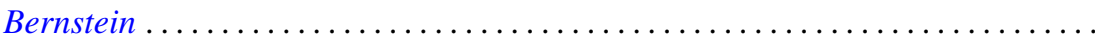

Jerry Grossman, On groups with specified lower central series quotients . .........

William H. Julian, Ray Mines, III and Fred Richman, Algebraic numbers, a constructive development . . . . . . . . . . . . . . . . . . . . . . .

Surjit Singh Khurana, A note on Radon-Nikodým theorem for finitely additive

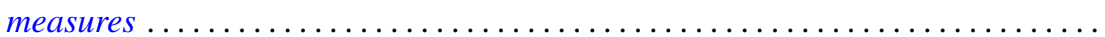

Garo K. Kiremidjian, A Nash-Moser-type implicit function theorem and nonlinear

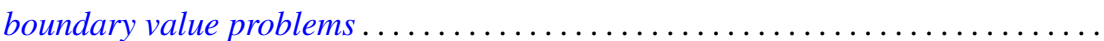

Ronald Jacob Leach, Coefficient estimates for certain multivalent functions ....

John Alan MacBain, Local and global bifurcation from normal eigenvalues. II . . 133

James A. MacDougall and Lowell G. Sweet, Three dimensional homogeneous algebras...

John Rowlay Martin, Fixed point sets of Peano continua ......

R. Daniel Mauldin, The boundedness of the Cantor-Bendixson order of some analytic sets...

Richard C. Metzler, Uniqueness of extensions of positive linear functions ..

Rodney V. Nillsen, Moment sequences obtained from restricted powers . .

Keiji Nishioka, Transcendental constants over the coefficient fields in differential elliptic function fields...

Gabriel Michael Miller Obi, An algebraic closed graph theorem

Richard Cranston Randell, Quotients of complete intersections by $\mathbf{C}^{*}$ actions . . 221

Bruce Reznick, Banach spaces which satisfy linear identities . .

Bennett Setzer, Elliptic curves over complex quadratic fields...

Arne Stray, A scheme for approximating bounded analytic functions on certain subsets of the unit disc.

Nicholas Th. Varopoulos, A remark on functions of bounded mean oscillation and bounded harmonic functions. Addendum to: "BMO functions and the

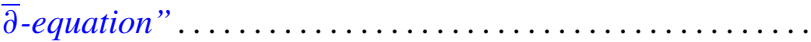

Charles Irvin Vinsonhaler, Torsion free abelian groups quasi-projective over their

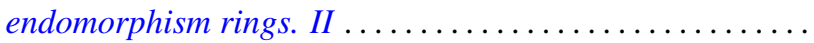

Thomas R. Wolf, Characters of $p^{\prime}$-degree in solvable groups ... 